lions, tigers and other big cats, but it ranges over the whole felid spectrum from the mighty Amur or Siberian tiger, largest of all the family, to the tiny rusty-spotted cat in India and Sri Lanka. Thirty-eight of the world's top specialists have contributed to this magnificent, spectacularly illustrated celebration of cats, 16 of them members of the Cat Specialist Group.

Starting, appropriately, with the evolution and biology of cats in general, with a special section on the awesome sabretooths, Great Cats looks at each species in turn with an account of their appearance, size, habitat, distribution, reproduction, social system and diet.

Paintings by Frank Knight show the 37 species in characteristic poses, and small maps provide a general picture of their range.

The contributors have produced highly-readable text, which should absorb the attention of all interested in the cats. Scientific terms are clearly explained. A chapter entitled 'How cats work' shows how specialized and efficient cats are as killing machines. Hunting techniques are well presented, and are matched by dramatic pictures.

Inevitably, the big cats, plus the puma (also big in size) and the lynx, get more coverage than the small because they have been easier to study - easier, but not easy. The highly secretive life of the small cats in forests, grasslands and deserts makes research a formidable challenge and little is known of their life in the wild.

Nevertheless, there is much to learn from the observations of small cats in captivity. It is a delight to see photographs of the rare Andean cat and the kodkod of southern Chile. But there isn't one of the Bornean bay cat-it is known from only a few skins and a mere handful of sightings.

An interesting section on wild cats and feral cats leads on to chapters on the place of cats in human history and culture, including a review of the evolution of domestic cats.

There are some gory pictures of victims of man-eating cats and discussion of the problem of reconciling conservation with the natural concern of local people living close to dangerous large predators. Unless this problem can be solved, or at least reduced to manageable proportions, the future of cats and other large animals appears gloomy.

The chapter on 'Cats in zoos' explains the efforts being made to ensure species survival in captivity. After taking a look at the growing need to manage wild populations, it predicts that wildlife reserves will become megazoos and concludes with the 'not too futuristic' scenario of tigers being tracked by satellites by way of injectable microchips carrying the animals genealogy and other biological information for integration with ecological, climatic and political variables in a centralized, map-linked data base. This data base would provide politicians and worldwide conservation leaders with information on which to formulate decisions about the economic growth of a country with due consideration of the impact on animal conservation.

The World Conservation Union's Chief Conservation Officer, Jeffrey McNeely, manages to be optimistic about the future of wild cats, even after detailing the survival problems they face. Let us hope that his optimism is justified, for the survival of the wild cats can only continue if the diverse animal and plant life on which they depend is conserved.

Preservation of the big cats is the best recipe for conserving biodiversity in their ranges.

There is a serious omission for a book of such high scientific quality-neither a bibliography nor references-but it is likely to be a long time before it can be matched, let alone bettered.

Peter Jackson.

\section{The Mammals of Arabia}

David L. Harrison and Paul J. J. Bates.

Harrison Zoological Museum, Sevenoaks, 1991, 354 pp., ISBN $0951731300, \mathrm{HB} £ 65$ or \$US120

The first edition of David Harrison's The Mammals of Arabia, published in three volumes from 1964 to 1972 , was indeed a 'first' in documenting the Arabian mammals in a comprehensive way. A reviewer of the first volume wrote that '... as a standard reference work on its subject it will undoubtedly last out the century'. He may not have envisaged that a substantially revised and updated edition would be needed to achieve this. But here it is, in a single volume of $\mathbf{3 5 0}$ pages replacing the 670 pages of the previous edition.

Although the geographical scope is unchanged, the title, as before, is a little misleading because the area covered is much more than the Arabian peninsula, extending northwards to include Israel, Jordan, Lebanon, Syria and Iraq. The 150 species dealt with, therefore, include a considerable number that are very peripheral to Arabia, such as the lynx and brown bear in Kurdistan and several other northern species like the badger and beech marten, which reach their southern boundary in the 
Mediterranean littoral of Israel. However, this is a useful bonus because there are no comprehensive modern works on the mammal faunas of these countries.

Several species have had to be added since the first edition, some new to science such as the Queen of Sheba's gazelle Gazella bilkis, the Arabian pipistrelle Pipistrellus arabicus and the Arabian white-toothed shrew Crocidura arabica; others newly recorded from Arabia, including Crocidura somalica and the lesser kudu, although the origin of the two recorded specimens of kudu, and the survival of the species in Arabia, are doubtful. For most species additional data are available, whether on distribution, behaviour, ecology etc., and all have been carefully documented, with sources. Its value for quick reference would have been enhanced, however, if topics such as habitat, food and reproduction had been given separate headings instead of being included under 'Remarks', which in some cases fill a column or more without paragraph breaks.

The reduction from the first edition has been achieved in a number of ways without detriment to the quality and the usefulness of the work. Descriptions of morphological details have been considerably condensed except where they are useful for the confirmation of identification in the more difficult groups; the maps and illustrations have been reduced in size without any loss of clarity; and the tables of measurements have been condensed by giving means and ranges rather than measurements of individual animals. The result is considerably greater clarity, enhanced by greatly enhanced layout and typography. Identification keys are given throughout and are much more clearly presented than before.

For the many species of larger mammals whose ranges have drastically declined in the last century, the decline is well documented in the text but it would have been helpful to distinguish between old and recent records on the maps.

As far as any volume can be described as definitive, this is the definitive reference on the mammals of this important and volatile region. It is a safer prediction now than it was in 1964 that this will 'last out the century' but even so at least two species have arrived too late for inclusion (a new species of mouse Apodemus hermonensis in Israel and the African mongoose Bdeogale crassicauda in Yemen), and no doubt there will be many more changes for better and worse to be documented in the future.

Gordon B. Corbet.

\section{Beyond Captive Breeding: Re-introducing Endangered Mammals to the Wild: Zoological Society of London Symposia 62 \\ J. H. W. Gipps (editor) Clarendon Press, Oxford, 1991, 284 pp., ISBN 019 8540191 , HB $£ 45.00$}

The release into the wild, for reintroduction, of captive-bred animals, with the ultimate aim of re-establishing populations, has become an area of keen interest in contemporary conservation. Beyond Captive Breeding discusses the advantages and limitations of this approach to conservation and establishes criteria for reintroductions and how best to carry them out. The volume contains 17 articles by 27 expert authors covering the theoretical and practical factors involved with reintroductions and includes case studies of scimitar-horned oryx, addax, black-footed ferret, red wolf and golden lion tamarin for illustration.

The contributors discuss the many biological and non-biological factors involved in the decision to proceed with a reintroduction effort, and suggest that this approach may be useful only for threatened species for which large areas of suitable habitat remain and for which adequate protection can be assured. In consequence, the greatest value of reintroductions may be in the habitat protection that they entail and in the public support for such projects that they can encourage. Liz Price.

\section{The Birds of Pakistan Volume 1 \\ T. I. Roberts \\ Oxford University Press, 1991, 598 pp., ISBN 019 $5774043, \mathrm{HB} £ 40$}

This large, informative work is the first of two volumes, covering 347 of a total of 660 species. Its style is now a rarity in the ornithological literature-a country avifauna in which the author not only provides detailed information on distribution and status (accompanied by 285 maps and a gazetteer), but also includes sections on identification, habits, breeding biology and vocalizations. Much of what is written is original, based on 30 years studying wildlife in Pakistan. Unlike the compressed style of most recent avifaunas, the accounts are frequently a delight to either dip into or browse through, especially those relating to nearendemic species such as western tragopan Tragopan melanocephalus, Syke's nightjar Caprimulgus mahrattensis and 